\title{
USO DO DOPPLER TRANSCRANIANO PARA MONITORIZAÇ̃̃O DO VASOESPASMO CEREBRAL SECUNDÁRIO À HEMORRAGIA SUBARACNÓIDE
}

*M. Radanovic, M. Scaff

Divisão de Clínica Neurológica do Hospital das Clínicas da FaculdadedeMedicina daUSP, S. Paulo-SP.

RESUMO - 0 vasoespasmo cerebral é responsável por aumento de morbidade e mortalidade em pacientes acometidos por hemorragia subaracnóide (HSA). Sua detecção de forma precoce e nãoinvasiva pelo método de Doppler transcraniano (DTC) pode contribuir para um melhor prognóstico destes doentes.

OBJETIVO. Discutir o uso do método de DTC para diagnóstico do vasoespasmo cerebral secundário à HSA espontânea.

Métodos. Foram analisados os registros de velocidade de fluxo em artérias intracranianas por DTC em 3 I pacientes com diagnóstico tomográfico de HSA, realizados seqüencialmente em conjunto com exame neurológico. Todos os pacientes (à exceção de um) eram portadores de aneurismas intracranianos.

REsULTADOS. Vasoespasmo clínico foi constatado em II pacientes ( $36,6 \%$ do total), com correspondente vasoespasmo ultrasonográfico presente em nove casos (82\%). Em três casos (33,3\%) as alterações de velocidade de fluxo precederam os sinais clínicos. Entre os 20 pacientes assintomáticos, 15 (75\%) apresentaram medidas de velocidade normais, havendo evidência de vasoespasmo sonográfico sem repercussão clínica em cinco (25\%). 0 índice de falso negativo na correlação entre exame clínico e DTC (sinais clínicos de vasoespasmo presentes, com velocidades de fluxo normais) foi de $18 \%$. No entanto, houve correspondência entre os achados clínicos e ultra-sonográficos em 26 dos 31 pacientes $(83,8 \%)$. Os valores de velocidades médias nas várias artérias e sua interpretação para cada subgrupo são discutidos com maiores detalhes.

Conclusão. 0 método de DTC é um auxiliar valioso na deteç̧ão pré-sintomática do vasoespasmo secundário à $H S A$, permitindo uma intervenção terapêutica mais precoce. Os dados obtidos neste trabalho coadunam-se com os diversos estudos publicados na literatura.

UnITRMOS: Isquemia cerebral transitória. Ultrasonografia. Diagnóstico.

\section{INTRODUÇÃo}

No paciente acometido por hemorragia subaracnóide (HSA), em especial quando secundária à ruptura de aneurismas saculares (derivados em sua maior parte das artérias do polígono de Willis), o vasoespasmo cerebral é o principal fator de aumento da morbidade e mortalidade, ao lado do ressangramento do aneurisma, sendo responsável por piora neurológica em cerca de $30 \%$ dos pacientes com esta doença' .0 espasmo arterial intracraniano geralmente se torna sintomático a partir do $3^{\circ}$ dia após

\footnotetext{
*Correspondência: R. CristianoViana, 163/92 Cep:05411-000-SãoPaulo-SP E-mail:mgomes@tecway.com.br
}

o sangramento, atingindo seu máximo entre $06^{\circ}$ e $10^{\circ}$ dias, com melhora após 12 dias $^{2}$. Dispomos atualmente de algumas opções terapêuticas que permitem ao paciente uma melhor evolução clínica, como a indução de hipertensão/hipervolemia, o uso de bloqueadores de canais de cálcio e a realização de angioplastia transluminal. 0 exame padrão para diagnóstico do vasoespasmo cerebral é a arteriografia cerebral, um método caro, invasivo e não isento de complicações, incluindo-se a piora do próprio vasoespasmo, não permitindo o diagnóstico precoce desta complicação ou o acompanhamento de sua evolução temporal, e tornando-a, muitas vezes, um diagnóstico de exclusão.

A introdução da técnica de estudo da velocidade de fluxo das artérias intracranianas pelo Doppler transcraniano (DTC) tornou possível este acesso não invasivo ao estudo da hemodinâmica cerebral, fornecendo parâmetros para o diagnóstico e acompanhamento da evolução temporal e resolução do vasoespasmo de forma segura, rápida e que pode ser realizado à beira do leito de forma seriada, não acrescentando nenhum risco a um paciente que muitas vezes já se encontra em situação crítica. $\mathrm{O}$ objetivo deste artigo é discutir o papel deste método auxiliar na monitorização de pacientes com HSA pela possibilidade de detecção precoce daqueles em risco para desenvolvimento de vasoespasmo e infarto cerebral associado, bem como seu papel auxiliar na programação terapêutica mais 
adequada ao paciente.

O primeiro registro feito por DTC foi realizado por Aaslid em $|98|^{3,4}$, sendo a metodologia desenvolvida pelo Departamento de Neurocirurgia da Universidade de Berna ${ }^{(5)}$, e desde então o método vem progressivamente se firmando como um valioso auxílio no diagnóstico de doenças vasculares cerebrais. $\mathrm{O}$ acesso aos vasos intracranianos é possível através de áreas em que o osso da calota craniana é fino ou através dos forames naturais (janelas acústicas), usando-se um transdutor de baixa freqüência $(2 \mathrm{mHz}$ ) que emite ondas de ultrasom de forma pulsátil. As janelas acústicas empregadas convencionalmente são a transtemporal (localizada acima do arco zigomático), transorbitária e transforaminal (forame magno), através das quais pode-se obter registros das velocidades de fluxo das artérias carótida interna $(\mathrm{ACl})$ intracraniana, cerebral anterior $(A C A)$, cerebral média $(A C M)$, cerebral posterior (ACP), comunicante anterior (ACOA), comunicante posterior $(A C o P)$, oftálmica $(A O)$, vertebral $(A V)$ e basilar (AB). Para uma correta identificação das artérias a partir dos sinais obtidos, são usados vários parâmetros, como a janela acústica utilizada, a orientação (angulação) do transdutor, a profundidade da amostra de sinal obtido, a direção do fluxo e o contorno da onda obtido após análise espectral.

Os achados de maior valor na interpretação do exame são a velocidade média $(\mathrm{Vm})$ de fluxo obtida no registro e o índice de pulsatilidade (IP), que é o grau de variabilidade da velocidade máxima de fluxo que ocorre durante as diferentes fases do ciclo cardíaco, refletindo a resistência periférica do órgão suprido pela artéria analisada (normalmente baixa no caso da circulação intracraniana). Os valores médios normais de velocidade de fluxo nas principais artérias são: $A C M$ - 4 I a 67 cm/seg; $A C A$ - 36-64 $\mathrm{cm} / \mathrm{seg} ; A C P$ - 3 I a $49 \mathrm{~cm} / \mathrm{seg} ; A C l-30$ a
54 cm/seg; $A V$ - 27 a 45 cm/seg; $A B$ - 80$110 \mathrm{~cm} / \mathrm{seg}^{(6)}$.

Algumas aplicações clínicas do método incluem: diagnóstico de morte encefálica ${ }^{7}$, estudo de malformações arteriovenosas ${ }^{8} \mathrm{e}$ acidentes vasculares cerebrais (caracterização de estenoses ou oclusões arteriais, detecção de êmbolos) $)^{9,10}$, monitorização de cirurgia de carótida ${ }^{11,12}$, teste da reserva vasomotora cerebral|'3, avaliação de traumatismo craniencefálico ${ }^{7}$, insuficiência vértebro-basilar ${ }^{14}$ e enxaqueca ${ }^{15}$. O propósito deste artigo é discutir o uso do DTC na monitorização do vasoespasmo pós- HSA.

\section{Métodos e Casuística}

Foram incluídos no estudo 3 I pacientes (19 mulheres e 12 homens), com idades entre 29 e 67 anos (média: 46,9 \pm II anos), admitidos no Pronto Socorro de Neurologia do Hospital das Clínicas da Faculdade de Medicina da Universidade de São Paulo com diagnóstico inicial de HSA feito por tomografia computadorizada (TC) de crânio. Os pacientes foram submetidos a exame neurológico realizado pelo mesmo examinador em dias alternados, durante 14 dias, a fim de detectar alterações compatíveis com o desenvolvimento de vasoespasmo cerebral, sendo que os casos em que se observou modificação em relação ao exame neurológico inicial foram reavaliados do ponto de vista metabólico e tomográfico, a fim de excluir outras causas de piora neurológica (distúrbios hidroeletrolítcos, ressangramento do aneurisma, desenvolvimento de hidrocefalia hipertensiva). Os pacientes foram avaliados por exames de DTC sucessivos neste período de I 4 dias ( 2 a 4 exames por paciente, com exceção de 7 pacientes que realizaram apenas um exame), perfazendo um total de 75 exames. Em todos os exames foram abordadas as janelas transtemporais, transorbitais (bilateralmente) e transforaminal, a fim de se tentar obter registros de velocidade de fluxo do maior número de artérias intracranianas. Todos os pacientes foram submetidos a exame angiográfico para pesquisa etiológica do sangramento (com exceção do caso 3 I que foi a óbito antes de realizar o exame), sendo detectados um ou mais aneurismas em 28 casos; dois pacientes apresentaram angiografia normal. Os exames angiográficos foram realizados precocemente, em geral I ou 2 dias após a admissão do paciente ao hospital, não tendo sido repetidos para confirmação do diagnóstico de vasoespasmo, tendo em vista o potencial de aumento da morbidade relacionado a este procedimento.

\section{Resultados}

A tabela I demonstra os resultados obtidos através das avaliações clínicas e por DTC seriadas realizadas nos pacientes. Vasoespasmo clínico foi constatado em II pacientes (36,6\% do total). Deste grupo, nove $(82 \%)$ apresentaram alterações de velocidade de fluxo registradas pelo DTC e em dois (I8\%) as medidas foram persistentemente normais. Quanto à distribuição das artérias acometidas, encontramos três casos com espasmo de ACM (média das velocidades de fluxo registradas: $21 \mathrm{l} \mathrm{cm} / \mathrm{seg}$ ), oito casos com espasmo de ACA (média das velocidades de fluxo: $100 \mathrm{~cm} / \mathrm{seg}$ ) e três casos com espasmo em $\mathrm{ACl}$ (média das velocidades: $152 \mathrm{~cm} / \mathrm{seg}$ ), sendo que em alguns pacientes havia sobreposição de vasos acometidos. Dos 20 pacientes que não desenvolveram vasoespasmo clínico, 15 (75\%) mostraram velocidades de fluxo normais em todos os exames de DTC realizados e cinco (25\%) apresentaram elevações compatíveis com diagnóstico ultra-sonográfico de vasoespasmo. A média das velocidades de fluxo no grupo sem vasoespasmo clínico e sonográfico foi de 68,4 \pm $15 \mathrm{~cm} / \mathrm{seg}$ para $A C M, 60 \pm 10,3 \mathrm{~cm} / \mathrm{seg}$ para $A C A$ e 53,6 $\pm 16,7 \mathrm{~cm} / \mathrm{seg}$ para $\mathrm{ACl}$. 


\section{Discussão}

Em 1984, Aaslid et al. descreveram a correlação entre a diminuição de diâmetro da ACM e aumento da velocidade de fluxo nesta artéria, de forma que pacientes com espasmo angiográfico de ACM apresentavam Vmentre 120 e $240 \mathrm{~cm} / \mathrm{seg}$, sendo que velocidades acima de $200 \mathrm{~cm} / \mathrm{seg}$ corresponderiam a um estreitamento arteriográfico superior a $50 \%{ }^{16}$. Esta correlação foi confirmada posteriormente em estudos realizados por diversos grupos ${ }^{17,18,19}$. As velocidades de fluxo aumentam progressivamente entre terceiro e oitavo dias após o sangramento, alcançando um pico entre 0 sétimo e décimo-quinto dias, podendo permanecer elevadas por quatro semanas ou mais. $\mathrm{O}$ aumento é, em geral, bilateral e acomete várias artérias, sendo usualmente maior ipsilateralmente ao aneurisma roto e nas regiões onde há maior quantidade de sangue no espaço subaracnóide ${ }^{20,21}$.

Pelo fato da velocidade de fluxo apresentar uma grande variabilidade individual, é mais adequado o uso de variações relativas da velocidade de fluxo, em lugar de seus valores absolutos. $O$ diagnóstico de vasoespasmo é feito, então, quando se registra um aumento de mais de $50 \%$ do valor inicial (feito nos primeiros três dias) da velocidade. Nos casos em que este valor inicial não é conhecido, assume-se uma Vm na ACM maior que $120 \mathrm{~cm} / \mathrm{seg}$ como indicativa de estreitamento arterial e uma Vm maior que $200 \mathrm{~cm} / \mathrm{seg}$ como altamente provável da existência de vasoespasmo angiográfico ${ }^{22}$. Estados de hiperfluxo global podem ser diferenciados de espasmo arterial através do índice hemisférico (formulado por Aaslid $^{26}$ e Lindegaard ${ }^{24}$, trata-se da razão $\checkmark A C M / v A C l$ ), que compensa parcialmente os efeitos da variação de fluxo, situando-se normalmente entre $1,7 \pm 0,4$, apresentando valores aumentados (em geral acima de 3) nos casos de espasmo em ACM. Os

Tabela I - Resultados das avaliações clínicas por DTC e angiografia nos pacientes com HSA.

\begin{tabular}{cc}
\hline Paciente & Vasoespasmo Clínico \\
1 & ausente \\
2 & ausente \\
3 & ausente \\
4 & ausente \\
5 & hemiplegia E \\
6 & NL \\
7 & hiperreflexia E \\
8 & afasia/hemiparesia D \\
9 & hemiparesia $\mathrm{E}$ \\
10 & ausente \\
11 & asente \\
12 & ausente \\
13 & ausente \\
14 & ausente \\
15 & ausente \\
16 & ausente \\
17 & ausente \\
18 & hemiparesia D \\
19 & ausente \\
20 & ausente \\
21 & ausente \\
22 & hemiparesia E \\
23 & ausente \\
24 & ausente \\
25 & hemiparesia E \\
26 & hemiparesia E \\
27 & hemiparesia D \\
28 & ausente \\
29 & ausente \\
30 & hemiparesia D \\
31 & hemiplegia E / coma
\end{tabular}

\begin{tabular}{|c|c|}
\hline DTC & Angiografia \\
\hline VEACMD & An ACoPD \\
\hline $\mathrm{NL}$ & An ACMD \\
\hline $\mathrm{NL}$ & $\mathrm{AnACoA}$ \\
\hline VEACMD & $\mathrm{AnACOA}$ \\
\hline VEACAD & $\mathrm{An} A O E$ \\
\hline An ACoPD & \\
\hline VEACIE & An ACoPE \\
\hline VE ACM bilateral & An ACoPE \\
\hline $\mathrm{NL}$ & An ACMD \\
\hline $\mathrm{NL}$ & An ACop E \\
\hline $\mathrm{NL}$ & $\mathrm{An} A \mathrm{Co} \mathrm{A}$ \\
\hline $\mathrm{NL}$ & An ACoA e ACM D \\
\hline $\mathrm{NL}$ & An ACoPE \\
\hline $\mathrm{NL}$ & An ACoPD \\
\hline VEACMD & An ACM D e ACoP E \\
\hline $\mathrm{NL}$ & $\mathrm{NL}$ \\
\hline $\mathrm{NL}$ & $\mathrm{AnAClE}$ \\
\hline VEACl e ACA D & $\mathrm{An} A C \mathrm{~A}$ \\
\hline $\mathrm{NL}$ & An ACME \\
\hline $\mathrm{NL}$ & $A n A C o A$ \\
\hline VEACAD & An ACM bilat \\
\hline VEACAD & $A n A C o A$ \\
\hline VEACAE & $\mathrm{AnACoA}$ \\
\hline $\mathrm{NL}$ & An Basilar \\
\hline $\mathrm{NL}$ & $\mathrm{AnACoA}$ \\
\hline VEACAE & $\mathrm{AnACoA}$ \\
\hline VEACAE & $\mathrm{AnACoA}$ \\
\hline $\mathrm{NL}$ & $\mathrm{AnACoA}$ \\
\hline $\mathrm{NL}$ & $\mathrm{NL}$ \\
\hline VEACME & An ACoP e ACME \\
\hline VEACM e ACA bilat & NR \\
\hline
\end{tabular}

Legenda: NL - normal; VE - vasoespasmo; ACM - artéria cerebral média; ACA - artéria cerebral anterior; $\mathrm{ACl}$ - artéria carótida interna; $\mathrm{ACOA}$ - artéria comunicante anterior; $A C o P$ - artéira comunicante pósterior; $A O$ - artéria oftálmica; An - aneurisma; D direita; E - esquerda; bilat - bilateral. NR - não realizado

critérios de interpretação da velocidade de fluxo em ACM estão expostos na tabela 2. A figura I representa um exemplo da onda espectral correspondente ao registro de Vm em ACM.

A sensibilidade e especificidade do método para este diagnóstico variam em diversos estudos, situando-se, respectivamente entre 56,8 a $85 \%$ e 85 a $100 \%^{23,24,25}$. Os seguintes fatores podem resultar num estudo falso-negativo: janela acústica inadequada, artefatos (clipe cirúrgico, por exemplo), variações anatômicas vasculares, oscilação de fatores hemodinâmicos, reológicos e metabólicos, presença de hipertensão intracraniana.

Em nossa casuística, o índice de falsonegativo na correlação entre exame clínico 
e DTC (presença de sinais clínicos de vasoespasmo com registros de velocidades de fluxo normais) foi de 18\%. No entanto, houve correspondência entre os achados clínicos e ultra-sonográficos em 26 dos 31 pacientes (83,8\%). Foi possível a detecção de espasmo sonográfico antes da instalação do déficit neurológico em três pacientes (correspondendo a 33,3\% dos casos de vasoespasmo), sendo que este índice de diagnóstico precoce pode ser aumentado se o exame de DTC for realizado diariamente.

O vasoespasmo na ACA proximal é mais difícil de ser definido por haver maior dificuldade técnica de registro do sinal, não ser um vaso terminal e pela maior variabilidade anatômica individual, levando a alterações de padrão de fluxo com maior freqüência ${ }^{17}$. Por este motivo, alguns autores consideram mais importante a variação da velocidade de fluxo observada num determinado período do tempo (quando esta ultrapassa $50 \%$ do valor inicial) do que seu valor absoluto na caracterização do espasmo de $\mathrm{ACA}^{23}$. Nos oito pacientes de nossa casuística que apresentaram espasmo de ACA, houve grande variabilidade nas velocidades de fluxo, sendo a mais baixa $74 \mathrm{~cm} /$ seg e a mais alta $152 \mathrm{~cm} / \mathrm{seg}$. Nos casos em que a velocidade registrada foi mais baixa, foi possível observar a ocorrência de aumentos de mais de duas vezes o valor inicial em períodos de 24 a 48 horas.

Correlação entre as alterações de velocidade e déficit isquêmico: Velocidades entre 120 e 140 cm/seg, em geral, não estão correlacionadas com a presença de déficit isquêmico, embora alguns pacientes com medidas de velocidade nesta faixa, ou mesmo normais, tenham apresentado evidência de infarto à TC de crânio, o que pode ser explicado pela presença de espasmo de ramos distais ${ }^{8,25}$, reduções globais do fluxo sangüíneo cerebral e/ou perda do mecanismo de auto-regulação cerebral, hiperemia

Tabela 2 - Critérios para o diagnóstico de espasmo de ACM ao Doppler transcraniano

\begin{tabular}{cccc}
\hline $\begin{array}{c}\text { Velocidade média (ACM) } \\
\text { entre } 100 \text { e } 120 \mathrm{~cm} / \mathrm{seg}\end{array}$ & $\begin{array}{c}\text { Razão vACM/vACI } \\
\text { grau de estenose }\end{array}$ & $\begin{array}{c}\text { Interpretação } \\
\text { elevacão não espećfica, } \\
\text { vasoespasmo leve } \\
\text { (ou distal) }\end{array}$ \\
$\begin{array}{c}\text { entre } 120 \text { e } 200 \mathrm{~cm} / \mathrm{seg} \\
>200 \mathrm{~cm} / \mathrm{seg}\end{array}$ & $3-6$ & $25 \%$ & \\
& $>6$ & $>50 \%$ & $\begin{array}{c}\text { vasoespasmo moderado } \\
\text { vasoespasmo grave, } \\
\text { em geral sintomático }\end{array}$ \\
\hline
\end{tabular}

Figura I - Onda espectral correspondente a registro da velocidade média $(\mathrm{Vm})$ de fluxo em ACM esquerda. Os números à esquerda representam, respectivamente, a profundidade do sinal $(50 \mathrm{~mm})$, a velocidade de pico sistólico $(167 \mathrm{~cm} / \mathrm{seg})$, a Vm $(126 \mathrm{~cm} /$ seg) e o índice de pulsatilidade $(0,54)$. A Vm obtida corresponde a vasoespasmo moderado.

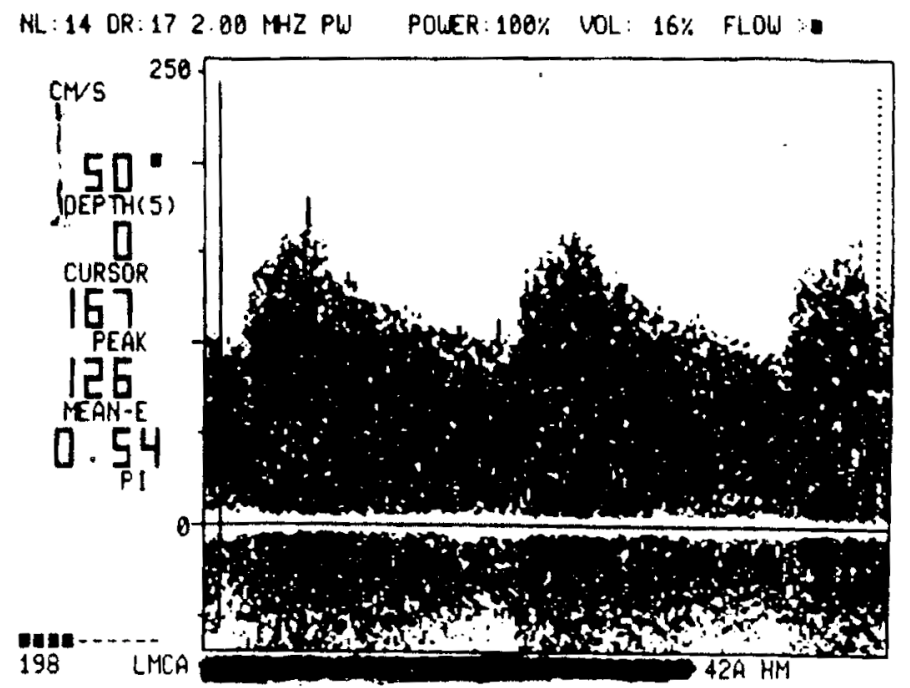

pós-operatória e a terapia hipertensiva. Harders et al. ${ }^{20}$, num grupo de 50 pacientes, observaram um risco de $25 \%$ para desenvolvimento de déficit isquêmico em pacientes com velocidades de fluxo acima de $140 \mathrm{~cm} / \mathrm{seg}$ na ACM. A mesma correlação entre o aumento da velocidade na ACM nos primeiros 10 dias após o sangramento, e risco aumentado para ocorrência de eventos isquêmicos foi registrada por Seiler et al. ${ }^{27}$ e Sekhar et al. ${ }^{19}$.

Velocidades acima de $200 \mathrm{~cm} / \mathrm{seg}$ na ACM estão fortemente associadas à isquemia cerebral, embora alguns pacientes possam ainda permanecer assintomáticos graças aos mecanismos compensatórios de fluxo. Grosset et al..$^{28}$ demonstraram que um aumento rápido da velocidade de fluxo na ACM (maior que $50 \mathrm{~cm} / \mathrm{seg} / 24 \mathrm{hs}$ ) é um valioso parâmetro preditivo da ocorrência de déficit isquêmico, documentado através do aparecimento de zonas de hipoperfusão ao SPECT, e constataram que mais de $60 \%$ dos pacientes com esta taxa de aumento da velocidade de fluxo apresentaram algum grau de déficit neurológico na sua evolução ${ }^{29}$. Assim, o registro precoce das alterações de velocidade nos primeiros sete dias após o sangramento 
permite detectar os pacientes com risco para o desenvolvimento de déficit isquêmico secundário ao vasoespasmo.

Em nossa casuística, nos três pacientes com vasoespasmo sintomático em ACM, dois apresentaram velocidades médias acima de $200 \mathrm{~cm} / \mathrm{seg}$ e em um caso as velocidades passaram três vezes o valor registrado inicialmente, situando-se acima de 160 $\mathrm{cm} / \mathrm{seg}$, o que configura vasospasmo moderado. Nos casos de vasospasmo sintomático em $A C A$, a média das velocidades foi de $100 \mathrm{~cm} / \mathrm{seg}$, enquanto no grupo assintomático foi de $60 \pm 10,3 \mathrm{~cm} / \mathrm{seg}$; nos casos de espasmo sintomático em $\mathrm{ACl}$, obtivemos uma média de velocidades de $152 \mathrm{~cm} / \mathrm{seg}$ contra 53,6 $\pm 16,7 \mathrm{~cm} / \mathrm{seg}$ nos indivíduos assintomáticos. Encontramos evidência de espasmo ultra-sonográfico sem repercussão clínica em cinco casos (correspondendo a 16,6\% do total de pacientes). Neste subgrupo, a média das velocidades foi de $156 \mathrm{~cm} / \mathrm{seg}$ nos três casos de espasmo em ACM e 83,5 cm/seg nos dois casos de espasmo de ACA, situando-se esses valores num nível intermediário em relação aos descritos acima.

O acompanhamento da variação das velocidades de fluxo arterial pós-HSA pode permitir uma melhor programação do momento mais adequado para cirurgia nos pacientes em que não foi possível a intervenção imediata ${ }^{30}$, possibilita a instituição precoce e monitorização da resposta à terapia hipertensiva hipervolêmica ${ }^{20}$ e auxilia a monitorização da eficácia da angioplastia para tratamento do vasoespasmo ${ }^{3 !}$.

\section{Conclusão}

O Doppler transcraniano é um método não-invasivo e barato, que permite o registro de velocidade de fluxo (e indiretamente, do diâmetro) das artérias basais cerebrais, sendo especialmente útil na identificação de vasoespasmo secundário à HSA. Exige do examinador certa experiência com a técnica, familiaridade com a anatomia vascular cerebral e alguns conceitos de hemodinâmica que permitam uma adequada interpretação dos dados obtidos. Algumas de suas limitações incluem a ausência de janela acústica em cerca de 10\% dos indivíduos, a impossibilidade de acesso aos vasos mais distais, a possibilidade de erros de interpretação decorrentes de variações anatômicas, e a susceptibilidade das velocidades registradas a sofrerem alteração em função da variação de alguns parâmetros fisiológicos que alteram a complexa hemodinâmica cerebral. A ACM é um vaso particularmente favorável para tal estudo, por seu fácil acesso e por estar freqüentemente acometida nos casos de vasoespasmo induzido por HSA.

A monitorização seriada mediante uso do DTC em pacientes com HSA permite a determinação pré-sintomática do vasoespasmo, o que é importante visto que os tratamentos disponíveis (hipertensão induzida, uso de bloqueadores de canais de cálcio e angioplastia transluminal) não são isentos de risco. Além disso, o método permite avaliar a resposta efetiva a esta terapêutica, tornando mais seguro e ágil o manejo das complicações. Os dados obtidos em nossa casuística fornecem um modelo da aplicação deste método, sendo que nossos resultados foram bastante similares aos descritos na literatura. Julgamos relevante o fato de que em 33,3\% dos pacientes que desenvolveram vasoespasmo sintomático, o diagnóstico foi possivel antes do aparecimento de sinais clínicos, o que certamente vem de encontro à necessidade de se instituir medidas terapêuticas o mais precocemente possível.

\section{SUMMARY}

USE OF TRANSCRANIAL DoppleR IN THE MONITORATION OF CEREBRAL VASOSPASM SECONDARY TO SUBARACHNOID HEMORRHAGE
Cerebral vasospasm accounts for an increased morbidity and mortality in patients with spontaneous subarachnoid hemorhage (SAH). Its early and non invasive detection by transcranial Doppler (TCD) may contribute to a better prognosis in such cases.

PURPose. To discuss the use of transcranial Doppler (TCD) in the detection of cerebral vasospasm secondary cerebral to spontaneous subarachnoid hemorrhage (SAH).

Methods. We analyzed the flow velocities registered by TCD in intracranial arteries in 31 patients with $\mathrm{SAH}$, performed sequentially, associated with neurological examinations. All patients (except one) had intracranial aneurysms.

Results. Clinical vasospasm was found in II patients (36,6\% from the total), with correspondent sonographic vasospasm present in 9 cases (82\%). In 3 cases (33.3\%) the flow velocity alterations preceded the clinical symptoms. Between the 20 asymptomatic patients, 15 (75\%) had normal flow velocity, with evidence of sonographic vasospasm without clinical repercussion in 5 (25\%). The index of false negatives in the correlation between clinical examination and TCD (symptomatic vasospasm with normal flow velocity records) was 18\%. However, there was correspondence between clinical and ultrasonographic findings in 26 of the 31 patients (83.8\%). The average flow velocity values in the various arteries and its interpretation for each subgroup are discussed with further details.

CONCLUSION. TCD is a valuable auxiliary method in the detection of cerebral vasospasm (even pre-symptomatic) secondary to $\mathrm{SAH}$, allowing a more precocious therapeutic intervention. Our results are similar to those described in the literature.

KEY-WORDS: Transient cerebral ischemia. Ultrasonography. Diagnosis. 


\section{Agradecimento}

Os autores agradecem ao Dr. Roberto Hirsch pelo ensino da técnica e colaboração na realização dos exames de DTC.

\section{ReferênCias Bibliográficas}

I. Torner, JC, Kassell, NF, Haley, EC. The timing of surgery and vasospasm. Neurosurg Clin North Am 1990; I: 335-47.

2. Smith, RR, Miller, JD Pathophysiology and clinical evaluation of subarachnoid hemorrhage. In: Youmans, JR ed. Neurological surgery. Philadelphia, W. B. Sauders, 1990.

3. Aaslid, R. The Doppler principle applied to measurement of blood flow velocity in cerebral arteries. In: Aaslid, R ed. Transcranial Doppler sonography, Wien-New York, Springer-Verlag, 1986; 22-38.

4. Aaslid, R, Markwalder, T, Nornes, H. Noninvasive transcranial Doppler ultrasound recording of flow velocity in basal cerebral arteries.J Neurosurg 1982; 57: 769-74.

5. Aaslid, R. Developments and principles of transcranial Doppler. In: Aaslid, R, Newell, DW eds. Transcranial Doppler, New York, Raven Press, 1992; . I-8.

6. Razumovsky, AY. Transcranial doppler ultrasonography in the intensive care unit. American Academy of Neurology Annual Meeting Neurologic Critical Care Workshop 1995: 25|-69-25|-74.

7. Newell, DW, Seiler, RW, Aaslid, R. Head injury and cerebral circulatory arrest. In: Aaslid, R, Newell, DW eds. Transcranial Doppler, New York, Raven Press, 1992; 109-21.

8. Hassler, W, Burger, R. Arteriovenous malformations. In: Aaslid, R, Newell, DW eds. Transcranial Doppler, New York, Raven Press, 1992; 125-35.

9. Mohr, JP, Hoffmann, M. Evaluation of stroke patients. In: Aaslid, R, Newell, DW eds. Transcranial Doppler, New York, Raven Press, 1992.; | 37-43

10. Spencer, MP. Detection of cerebral arterial emboli. In: Aaslid, R, Newell, DW eds. Trans- cranial Doppler, New York, Raven Press, 1992; 215-30.

I I. Jansen, C, Vriens, EM, Eikelboom, BC et al. Carotid endarterectomy with Transcranial Doppler and Electroencephalographic monitoring. A prospective study in 130 operations. Stroke 1993; 24:. 665-9.

12. Steiger, H.J. Monitoring for carotid surgery. In: Aaslid, R, Newell, DW eds. Transcranial Doppler, New York, Raven Press, 1992; 197-205.

13. Ringelstein, EB, Otis, SM Physiological testing of vasomotor reserve. In: Aaslid, R, Newell, DW eds. Transcranial Doppler, New York, Raven Press, 1992;.83-99.

I4. Kinsella LJ, Feldmann E, Brooks JM. The clinical utility of transcranial Doppler ultrasound in suspected vertebrobasilar ischemia. J Neuroimag 1993;3: I15-22.

I5. Friberg, L, Olsen, J, Iversen, HKet al. Migraine pain associated with middle cerebral artery dilatation: reversa by sumatriptan. Lancet 1991; 338: 13-7

16. Aaslid, R, Huber, P, Nornes, H. Evaluation of cerebrovascular spasm with transcranial Doppler ultrasound. J Neurosurg 1984; 60: 37-4l.

17. Grosset, DG, Straiton, J, McDonald, I, Bullock, R. Angiographic and Doppler diagnosis of cerebral arterial vasospasm following subarachnoid haemorrhage. Br J Neurosurg 1993; 6: 291-8.

18. Newell, DW, Grady, MS, Eskridge, JM, Winn, HR. Distribution of angiographic vasospasm after subarachnoid hemorrhage: implications for diagnosis by transcranial Doppler ultrasonography. Neurosurgery 1990;27: 574-7.

19. Sekhar, LN, Wechsler, LR, Yonas, $\mathrm{H}$ et al. Value of transcranial Doppler examination in the diagnosis of cerebral vasospasm after subarachnoid hemorrhage. Neurosurgery 1988; 22: 8|3-2|.

20. Harders, A, Gilsbach, JM. Time course of blood velocity changes related to vasospasm in the circle of Willis measured by transcranial Doppler ultrasound. J Neurosurg 1987; 66: 7|8-28.

2I. Hutchinson, K, Weir, B. Transcranial Doppler studies in aneurysm patients. Can J Neurol Sci 1989; 16:411-6

22. Newell, DW, Winn, HR. Evaluation of vasospasm using transcranial Doppler. Contemp Neurosurgery 1989; 1 1: 1 -6.
23. Grolimund, P, Seiler, RW, Aaslid, R et al. Evaluation of cerebrovascular disease by combines extracranial and transcranial Doppler sonography. Stroke 1987; 18: 10 | 8-24.

24. Lindegaard, KF, Nornes, H, Bakke, SJ et al. Cerebral vasospasm after subarachnoid hemorrhage investigated by means of transcrania Doppler ultrasound. Acta Neurochir (Suppl) 1988; 42: 8I-4

25. Sloan, MA, Haley, Jr., EC, Kassell, NF et al. Sensivity and specificity of transcranial Doppler ultrasonography in the diagnosis of vasospasm following subarachnoid hemorrhage. Neurology 1989; 39: 15।4-8.

26. Aaslid, R, Huber, P, Nornes, H. A transcranial Doppler method in the evaluation of cerebrovascular spasm. Neuroradiology 1986; 28: 11-6.

27. Seiler, RW, Grolimund, P, Aaslid, R et al. Cerebral vasospasm evaluated by transcranial ultrasound correlated with clinical grade and CT-visualized subarachnoid hemorrhage. J Neurosurg 1986; 64: 594-600

28. Grosset, DG, Straiton, J, Du Trevou, M, Bullock, R. Prediction of symptomatic vasospasm after subarachnoid hemorrhage by rapidly increasing transcranial Doppler velocity and cerebral blood flow changes. Stroke 1992; 23: 674-9.

29. Grosset, DG, Straiton, J, McDonald, let al. Use of transcranial Doppler sonography to predict development of a delayed ischemic deficit after subarachnoid hemorrhage. I Neurosurg 1993; 78: 183-7.

30. Sloan, MA. Detection of vasospasm following subarachnoid hemorrhage. In: Babikian, VL, Wechsler, LR eds. Transcranial Doppler Sonography, St Louis: Mosby 1993; I05-27.

31. Hurst, RW, Schnee, C, Raps, EC et al. Role of Transcranial Doppler in neuroradiological trea tment of intracranial vasospasm. Stroke 1993 24: 299-303.

Artigo recebido: 10/09/1999

Aceito para publicação: 18/07/2000 